\title{
Dry granular avalanche impact force on a rigid wall of semi-infinite height
}

\author{
Adel Albaba ${ }^{1,2, \star}$, Stéphane Lambert ${ }^{1, \star \star}$, and Thierry Faug ${ }^{1, \star \star \star}$ \\ ${ }^{1}$ Univ. Grenoble-Alpes, Irstea, ETGR, 38402 St-Martin d'Hères, France \\ ${ }^{2}$ Berne University of Applied Sciences, School of Agricultural, Forest and Food Science HAFL, Länggasse 85, CH-3052 Zollikofen, \\ Switzerland
}

\begin{abstract}
The present paper tackles the problem of the impact of a dry granular avalanche-flow on a rigid wall of semi-infinite height. An analytic force model based on depth-averaged shock theory is proposed to describe the flow-wall interaction and the resulting impact force on the wall. Provided that the analytic force model is fed with the incoming flow conditions regarding thickness, velocity and density, all averaged over a certain distance downstream of the undisturbed incoming flow, it reproduces very well the time history of the impact force actually measured by detailed discrete element simulations, for a wide range of slope angles.
\end{abstract}

\section{Introduction}

The mitigation of natural hazards related to landslides and avalanche requires a thorough understanding of the interaction between large-scale granular flows and civil engineering structures. This includes the development of new knowledge on the kinematics of free-surface granular flows-down inclines-disturbed by the presence of obstacles, as well as on the force imparted to the obstacle. One interesting approach is to develop depth-averaged analytic solutions, useful for engineers in charge of the design of structures subjected to landslides and avalanches, as recently reviewed in [1]. The present paper describes a contribution to this topic, showing how an analytic model based on depth-averaged shock theory can capture the impact force of a granular avalanche-flow on a rigid wall which cannot be overtopped (wall of semi-infinite height), provided that the relevant depth-averaged properties of the incoming flow are used as input parameters.

Numerical simulations using the discrete element method (DEM) to mimic the impact of a granular avalanche-flow on a rigid wall are briefly described in section 2. An analytic force model based on depthaveraged shock theory is presented in section 3. Section 4 addresses the main outcomes of our study regarding the cross-comparison between the depth-averaged analytic model and the results from DEM simulations. Finally, section 5 concludes on the need for future research.

\section{DEM simulations}

The impact of the granular avalanche-flow on the rigid wall is simulated with the help of DEM, using the open-

\footnotetext{
^e-mail: adel.albaba@bfh.ch

$\star \star$ e-mail: stephane.lambert@irstea.fr

$\star \star \star$ e-mail: thierry.faug@irstea.fr
}

source YADE software [2]. Samples were prepared using clumped particles ranging from 12.5 to $25 \mathrm{~mm}$ in diameter, interacting through a linear-dashpot model with a Mohr-Coulomb failure criterion. Particles were flowing in an inclined chute with different angles ranging from 30 to 55 degrees which is bound by a rigid wall at the bottom. Much more detail on the parameters used in the DEM simulations, as well as on the validation of the DEM model on existing laboratory tests on dry granular avalanche impact on a rigid wall, can be found in [3].

Figure 1 shows series of snapshots of the granular flow evolution. It starts with the impact of a very dilute front that is too discrete in nature and can not be represented by a continuum model. Afterwards, a more dense incoming flow arrives and a distinguished dead zone (gray) can be observed. That zone is followed by a green zone representing the part where the incoming flow conditions (height, velocity ..etc) are averaged and then used in the analytic model as inputs. Different inclination angles (Fig.1 top and bottom) leads to different evolving and final dead zone shape, basal friction contribution and propagating wave speed. The objective of the depth-averaged analytic model is to determine the evolution of impact force with time including all the complexities arising from different inclination angles.

\section{Depth-averaged analytic model}

The flow of granular materials down the incline, of slope $\alpha$ and constant width $w$, can be described by its thickness $h_{1}$, and its depth-averaged quantities, namely the velocity $\bar{u}_{1}=\frac{1}{h} \int_{0}^{h_{1}} u(z) d z$ and the volume fraction $\phi_{1}$. The avalanche-flow then impacts a rigid wall normal to the bottom and of semi-infinite height, thus preventing overflow downstream of the wall. As a result, the incoming flow of granular materials is pushed upstream the wall, which 

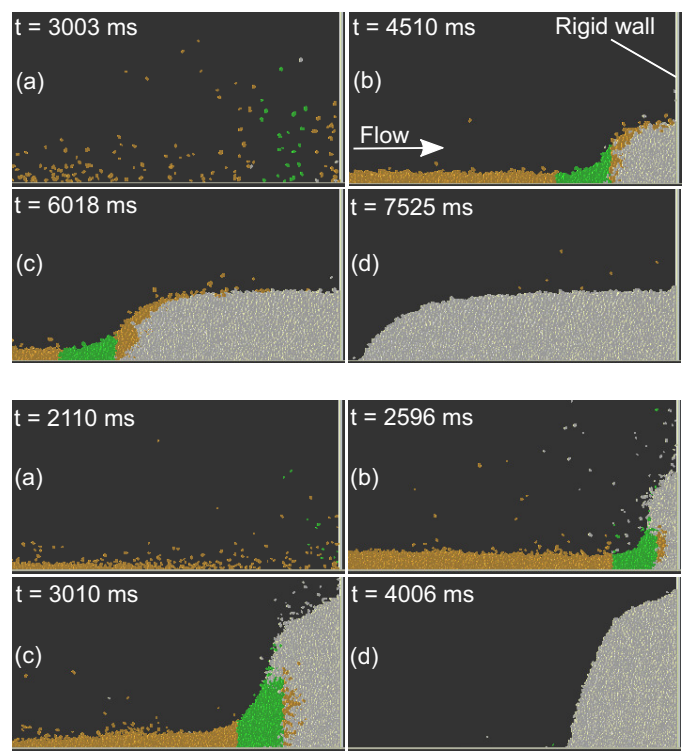

Figure 1. Impact of the avalanche-flow with the wall (a), formation (b), propagation of the granular bore (c), and final arrest of the flow (d), as simulated by DEM for two slopes angles $\alpha=30^{\circ}$ (top panel) and $\alpha=42.5^{\circ}$ (bottom panel). Orange: flowing particles, green: flowing parameters sampling zone, gray: dead zone particles

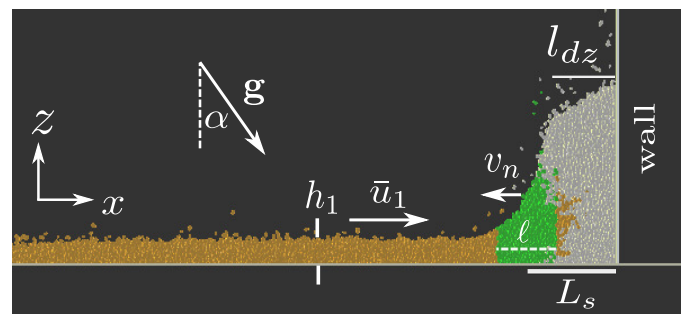

Figure 2. Granular bore forming upstream of a rigid wall impacted by a flow of granular materials: DEM simulations (example for $\alpha=40^{\circ}$ ) and notation

produces a granular shock-wave propagating upstream, the grains trapped between the wave and the wall forming a stagnant zone. A general picture of this granular flow-wall interaction is displayed in Fig. 2 showing a snapshot extracted from the DEM simulations presented in section 2.

\subsection{Simple description of the granular bore}

We propose to describe the interface between the incoming granular flow and the stagnant zone formed upstream of the wall using depth-averaged shock theory. As depicted in the sketch in Fig. 3, we define the following variables: the filling height $h_{d z}$ against the wall, the length $l_{d z}$ of the dead zone, the jump height $h_{2}$, the mean velocity $\bar{u}_{2}$ within the jump, the volume fraction $\phi_{2}$ and $v_{n}$ the propagation speed of the wave. The dead zone is defined by a volume fraction $\phi_{d z}$ corresponding to static close packing $\left(\phi_{d z} \sim\right.$ $0.64)$ and a mean velocity $u_{d z}=0$. Also we consider that $\phi_{2}$ (volume fraction within the shock) is equal to $\phi_{d z}$ (mean volume fraction inside the dead zone). Because we assume

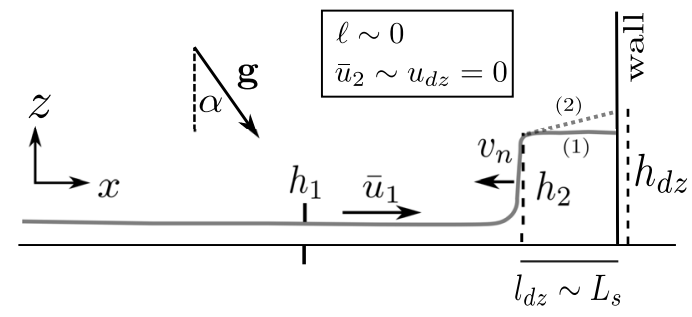

Figure 3. Sketch of a granular bore for the derivation of the depth-averaged shock model

that $\ell \sim 0$, we do not describe the variation of $\phi$ from $\phi_{1}$ to $\phi_{2}$ but rather consider a strong shock-wave in density.

The integral forms of mass and momentum conservation equations across the compressible jump are given by the following relations (see more detail in [4]):

$$
\left[\left[\rho_{P} \phi h\left(\mathbf{u}-\mathbf{v}_{\mathbf{n}}\right) \cdot \mathbf{n}\right]\right] w=0,
$$

$$
\left[\left[\rho_{P} \phi h \mathbf{u}\left(\mathbf{u}-\mathbf{v}_{\mathbf{n}}\right) \cdot \mathbf{n}+\frac{1}{2} \rho_{P} \phi g h^{2} \cos \alpha \mathbf{n}\right]\right] w=\mathbf{S} .
$$

The jump bracket $[[f]]=f_{1}-f_{2}$ denotes the difference between the enclosed function $f$ on the forward and rearward sides of the singular surface, following the notation commonly used in the literature about granular shocks $[5,6]$. Hydrostatic pressure distribution is assumed at each side of the jump $\left(k=\sigma_{x x} / \sigma_{z z}=1\right.$ with $\left.\sigma_{z z}=\rho g(h-z) \cos \alpha\right)$, as well as uniform velocity profiles $\left(\beta=\bar{u}^{2} / \bar{u}^{2} \sim 1\right)$. The density of the granular fluid is $\rho_{P} \phi$, where $\rho_{P}$ is the particle density. A detailed discussion on considering values of $k$ and $\beta$ slightly different from 1 (either upstream or downstream the shock) is given in $[1,4]$ and some other references therein. In the momentum equation, assuming that the jump volume shrinks into a singular surface $(\ell \sim 0)$ yields $\mathbf{S}=0$. In other words, the volume and surface forces acting over the finite length of the jump are neglected here, though a recent experimental study dedicated to standing jumps formed in granular flows revealed the importance of those forces under certain circumstances when the jumps become diffuse [4]. This crucial assumption will be further discussed while concluding the present paper.

In the problem of granular impact against a wall of semi-infinite height, the normal velocity at the outgoing section of the jump has to satisfy the velocity continuity, which means that $\overline{\mathbf{u}}_{\mathbf{2}} \cdot \mathbf{n}=u_{d z}=0$. From mass conservation, it yields an expression for the velocity $v_{n}$ at which the granular jump propagates upstream:

$$
v_{n}=-\frac{\left(\overline{\mathbf{u}}_{\mathbf{1}} \cdot \mathbf{n}\right) \mathbf{n}}{\frac{\phi_{2}}{\phi_{1}} \frac{h_{2}}{h_{1}}-1} .
$$

Note that the jump height $h_{2}$ is by construction smaller or equal to $h_{d z}$, as drawn in Fig. 3. $h_{2}$ can be predicted by injecting the relation for $v_{n}$ in the momentum conservation equation across the shock (Eq. 2), which after some rearrangements takes the following form:

$$
\left(\frac{h_{2}}{h_{1}}\right)^{3}+A\left(\frac{h_{2}}{h_{1}}\right)^{2}+B\left(\frac{h_{2}}{h_{1}}\right)+C=0,
$$


where $A=1 / \lambda_{\phi}, B=-\left(1+2 F r_{1}^{2}\right) / \lambda_{\phi}$, and $C=1 / \lambda_{\phi}^{2}$. We have defined here the density ratio $\lambda_{\phi}=\phi_{2} / \phi_{1}$ and the Froude number of the incoming flow $F r_{1}=$ $\bar{u}_{1} / \sqrt{g h_{1} \cos \alpha}$. This equation is a cubic equation that can be solved by Cardano's method.

\subsection{Impact force imparted to the rigid wall}

Once the grains start piling-up against the wall, the force imparted to the rigid wall is given by the apparent weight of the grains squeezed between the incoming flow and the wall. The interface between the incoming flow and the grains trapped against the wall is modeled by a strong compressible jump (see Sec. 3.1). Let's write the static equilibrium for the dead zone:

$$
\Sigma F_{e x t}=F_{s}+W_{d z} \sin \alpha-\mu_{d z} W_{d z} \cos \alpha+R=0 .
$$

The following forces are defined: $F_{s}$ is the force of the strong jump exerted on the dead zone, $W_{d z} \sin \alpha$ is the weight of the dead zone along the slope, $\mu_{d z} W_{d z} \cos \alpha$ is the resistive friction force (where $\mu_{d z}$ denotes the Coulomb friction coefficient) and $R$ is the reaction of the wall. The total force on the wall is given by the following expression:

$$
F_{n}=-R=F_{s}+W_{d z} \cos \alpha\left(\tan \alpha-\mu_{d z}\right) \text {. }
$$

The weight of the dead zone is:

$$
W_{d z}=\rho_{P} \phi_{F} g c_{d z} l_{d z} h_{d z} w,
$$

where $l_{d z}$ is the dead zone length and $c_{d z}$ a shape coefficient. The distance $L_{s}$ at which the granular wave is located at time $t+d t$ can be estimated from the value at time $t$ using $L_{s}(t+d t)=L_{s}(t)+v_{n} d t$. In practice, it is mostly likely that the propagation speed is actually smaller than the theoretical value for $v_{n}$ because of some dissipation (by friction and collisions between grains). However, if we assume that $v_{n}$ matches the speed at which the material piles-up against the wall, we have: $l_{d z} \sim L_{s} . L_{s}$ should then be always an overestimation of $l_{d z}$.

Moreover, it is worthwhile to stress that the jump height $h_{2}$ is not the run-up height $h_{d z}$ on the wall. $h_{d z}$ takes the following form: $h_{d z}(t+d t)=\max \left(h_{2}(t), h_{2}(t+d t)\right)+$ $h_{+}$, where $h_{+}$is an additional contribution to the run-up, as shown in the sketch on the bottom panel of Fig. 3. Predicting $h_{+}$remains a challenging question. There exists a time beyond which the jump height $h_{2}$, derived from shock theory, starts vanishing once the incoming flow starts dying. We then consider the maximum between $h_{2}(t+d t)$ and $h_{2}(t)$ because the filling height $h_{d z}=h_{2}+h_{+}$cannot decrease. Note that $c_{d z}>2$ if $h_{+}=h_{d z}-h_{2}$ is not nil, while $c_{d z}=2$ if $h_{d z}=h_{2}$. The latter situation corresponds to a dead zone that is nearly rectangular-see case (1) drawn in the sketch on bottom panel of Fig. 3, and may happen if the slope $\alpha$ is lower than a typical friction angle $\varphi$ associated with quasi-static deformations. The former situation holds for a dead zone being trapezoidal—see case (2) in the sketch on bottom panel of Fig. 3, and may occur when the slope $\alpha$ is greater than $\varphi$. In this case, the slope of the deposit is nearly equal to $\varphi$.

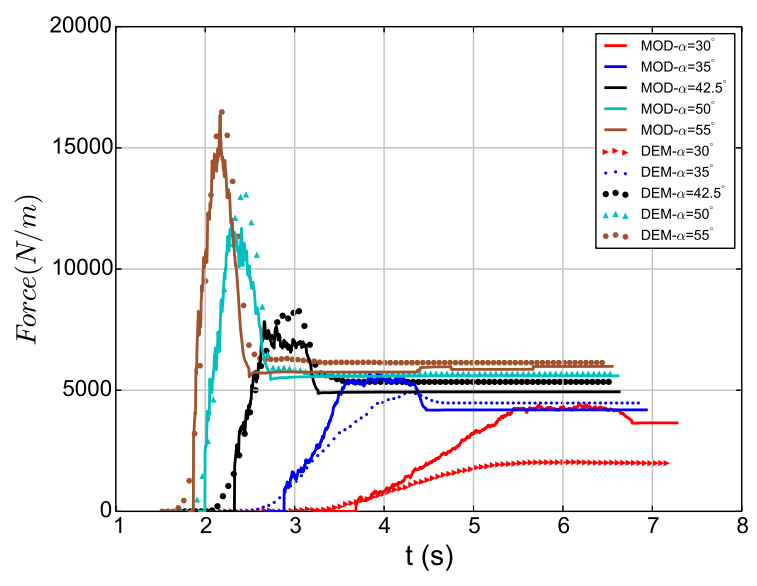

Figure 4. Temporal force signal on the rigid wall: prediction of the depth-averaged shock model (Eq. 6 fed with Eqs. 7 and 9, continuous lines) versus the force actually measured in DEM simulations (dots), for different slope angles $\alpha$

Finally, in the DEM simulations described at Sec. 2 a finite-sized mass $m_{\text {tot }}$ of material is initially released, which means that the mass of the dead zone is limited to this value $m_{t o t}: \rho_{P} \phi_{d z} c_{d z} l_{d z} h_{d z} w<m_{t o t}$. For given values of $h_{d z}, \phi_{d z}$ and $c_{d z}$, this yields a limit value for $l_{d z}$, itself approximated by $L_{s}$. In other words, the weight of the dead zone is restricted to the maximum value $m_{t o t} g$.

The force of the incoming flow on the dead zone can be predicted from the integral form of momentum conservation across the shock formed between the incoming flow and the dead zone (the forces acting over the finite length $\ell$ of the dead zone are still neglected here):

$$
\left[\left[\left[\rho_{P} \phi h \mathbf{u}\left(\mathbf{u}-\mathbf{v}_{\mathbf{n}}\right) \cdot \mathbf{n}\right]\right]-\left[\left[\int_{0}^{h} \sigma_{x}\right]\right] \mathbf{n}\right] w=0 .
$$

Under the assumption of hydrostatic pressure distribution for the incoming granular flow, this yields:

$$
F_{s}=\rho_{P} \phi_{1} u_{1}^{2} h_{1} w\left(1+\frac{1}{\frac{\phi_{2}}{\phi_{1}} \frac{h_{2}}{h_{1}}-1}+\frac{1}{2 F r_{1}^{2}}\right)
$$

Equation 6, fed with Eqs. 7 and 9, enables the estimation of the total force on the rigid wall, which is compared to the impact force actually measured in the DEM simulations in the next section.

\section{Depth-averaged model versus DEM}

A number of calculations were done to test the depthaveraged analytic force model presented in Sec. 3 and the various assumptions made. A full description of these tests will be given in a forthcoming paper. A key result is that the model was not able to capture the impact force actually measured in the DEM simulations if the properties of the incoming flow, taken at a singular surface before the jump, were used to feed the model. However, a good agreement could be found-over the whole range of slope 


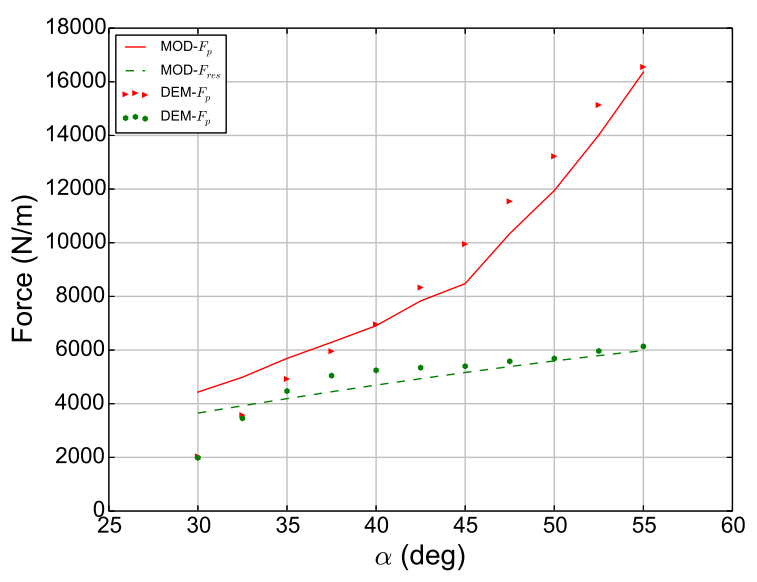

Figure 5. Peak and residual forces versus $\alpha$ : comparison between the shock model and the force measured in DEM

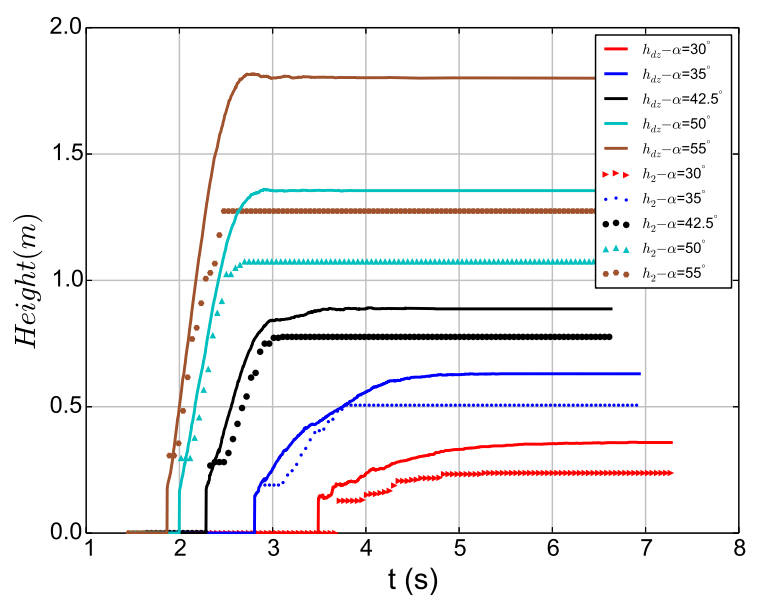

Figure 6. Evolution over time of the jump height $h_{2}$ predicted by the shock model (dots) and of the run-up $h_{d z}$ measured in DEM (continuous line)

angles tested, by feeding the force model with the incoming flow properties averaged over a certain region, starting from the beginning of the jump and stopping after a distance $15 d_{50}$ along the slope (see the green-colored regions on Fig. 1). These results are shown in Fig. 4.

The agreement is nearly perfect for the highest slope angles $\alpha$ but becomes less good while decreasing $\alpha$. Note that there always exists - whatever the slope - a time window (until and even after the peak for the highest $\alpha$ ) during which the increase of $F$ with time is very well captured by the analytic force model. The agreement is less good after this time window because the strong compressible shock does not longer exist but is largely damped by energy dissipation caused by friction and taking place along the slope. There is then a transition towards a much more diffuse jump, leading to the formation of a final deposit of characteristic shape. Of course this complicated physics related to a fluid-to-solid transition is not considered in the shock model and prevails at the lowest $\alpha$. As a result, the peak and residual forces cannot be well captured at the lowest $\alpha$, as depicted in Fig. 5 that shows the peak force $\left(F_{\text {peak }}\right)$ and the residual force $\left(F_{\text {res }}\right)$ extracted from the temporal signals displayed in Fig. 5.

As already discussed in section 3.1, the (saturated) height of the granular bore is - by construction - an underestimate of the run-up height $h_{d z}$ on the wall. This is confirmed by Fig. 6 showing the $h_{d z}$ actually in DEM and the jump height $h_{2}$ predicted by the shock model. Interestingly, the depth-gradients $\partial h_{d z} / \partial t$ and $\partial h_{2} / \partial t$ are very similar, thus probing the relevance of describing the impact by the shock model presented in Sec. 3 .

\section{Discussion and conclusion}

The present paper briefly presented DEM simulations of the impact of a dry granular avalanche-flow on a rigid wall. An analytic force model based on depth-averaged shock theory for compressible fluids was derived. This model was able to capture the evolution of the impact force with time actually measured in DEM if it was fed with the incoming flow properties averaged over a certain distance (equal to $15 d_{50}$ ) independent of the slope angle. This result shows that there exists a relevant length-scale, namely the jump length along the slope, relevant to the problem. The assumption $\ell \sim 0$ appears to be too strong and will need further investigation in the future. A detailed description of both the DEM simulations and the depth-averaged force model, as well as an extended description of the tests made to compare the analytic force model to the DEM results, are addressed in a paper currently under preparation.

\section{Acknowledgments}

TF is grateful to funding by the People Programme (MC Actions) of the EU 7th FP under REA Grant Agreement No. 622899 (FP7-PEOPLE-2013-IOF, GRAINPACT).

\section{References}

[1] Faug T., Physical Review E 92, 062310 (2015)

[2] https://yade-dem.org/doc/

[3] A. Albaba, S. Lambert, F. Nicot, B. Chareyre, Granular Matter 17(5), 603 (2015)

[4] T. Faug, P. Childs, E. Wyburn, I. Einav, Physics of Fluids 27(7), 073304 (2015)

[5] K.M. Hákonardóttir, A.J. Hogg, Physics of Fluids 17(7), 077101 (2005)

[6] J.M.N.T. Gray, X. Cui, Journal of Fluid Mechanics 579, 113 (2007) 